\title{
Epstein-Barr Virus-Associated Smooth Muscle Tumours after Transplantation, Infection with Human Immunodeficiency Virus and Congenital Immunodeficiency Syndromes
}

\author{
Kais Hussein ${ }^{a} \quad$ Britta Maecker-Kolhoffc, d Frank Donnerstag ${ }^{b} \quad$ Florian Laenger $^{a}$ d \\ Hans Kreipe $^{\mathrm{a}}$ Danny Jonigk ${ }^{\mathrm{a}, \mathrm{d}}$ \\ Institutes of a Pathology and ${ }^{\mathrm{b}}$ Neuroradiology, ${ }^{\mathrm{c}}$ Department of Paediatric Haematology and Oncology, and \\ ${ }^{\mathrm{d}}$ Integrated Research and Treatment Center Transplantation (IFB-Tx), Hannover Medical School, Hannover, Germany
}

\section{Key Words}

Smooth muscle tumours - Epstein-Barr virus .

Immunosuppression

\begin{abstract}
Smooth muscle tumours (SMT) after transplantation (PTSMT) or associated with congenital immunodeficiency syndromes (CI-SMT) and human immunodeficiency virus (HIV-SMT) are rare. The majority of PTSMT and CI-SMT are associated with Epstein-Barr virus (EBV), while some HIV-SMT can be EBVnegative. SMT in immunodeficient states may present with unspecific symptoms which are mainly related to tumour localisation. In PTSMT, $>50 \%$ of tumours manifest in the liver/ transplant liver, but in general PTSMT, HIV-SMT and CI-SMT can occur at any site as single or multiple tumours. Multiple tumour manifestations do not define metastatic disease as PTSMT can occur synchronously and/or metachronously. PTSMT can originate from the recipient as well as from the donor. Morphologically, most tumours, in particular PTSMT, lack marked histological atypia or tumour necrosis, while some HIV-SMT and CI-SMT can present as sarcoma-like variants, but histomorphology does not predict clinical aggressiveness or tumourbiological behaviour. In PTSMT, surgery and reduced immunosuppression show comparable overall
\end{abstract}

survival rates, while poor prognosis is mainly associated with intracranial manifestation and non-resectable tumours. In HIV-SMT and CI-SMT, surgery should be performed. In all 3 tumour types, adverse prognosis is mainly related to comorbidities associated with immunosuppression but not with the extent of histological atypia or tumour size.

Copyright $\odot 2013$ S. Karger AG, Basel

\section{Pathogenesis, Risk Factors and Molecular Pathology}

Sustained acquired or congenital immunosuppression is associated with an increased risk of developing neoplasms, in particular, Epstein-Barr virus (EBV)-associated diseases such as post-transplant lymphoproliferative disorder (PTLD) [1]. While PTLD can occur in up to $10 \%$ of transplant patients, EBV-positive post-transplant smooth muscle tumours (PTSMT) are rare $(<1 \%$ of patients) [1,2]. EBV-positive smooth muscle tumours (SMT) are not specific for transplant patients but are associated with any patients on long-term immunosuppression. Similar tumours can manifest in patients who suffer from human immunodeficiency virus (HIV) infection (HIV-SMT) or congenital immunodeficiency syndromes (CI-SMT) [3-11]. Among HIV patients and pa-

\section{KARGER}

(c) 2013 S. Karger AG, Basel

1015-2008/13/0806-0297\$38.00/0

E-Mail karger@karger.com

www.karger.com/pat
Kais Hussein, MD

Institute of Pathology, Hannover Medical School

Carl-Neuberg-Strasse 1

DE-30625 Hannover (Germany)

E-Mail hussein.kais@mh-hannover.de 
tients with rare congenital defects, the exact frequency of tumour manifestation is not known, but is most likely $<5 \%$.

Until now, no clear risk factors could be identified. There is no gender-specific risk increase regarding any of the three tumour types [2-11]. In transplant patients, the type of immunosuppressive drug, the transplant organ and the manifestation of PTLD are not associated with PTSMT manifestation [2]. About $60 \%$ of PTSMT can be found in kidney-transplant patients but it has to be taken into account that this is the most frequently transplanted organ [2]. In contrast to PTLD, PTSMT are late complications (median 48 months after transplantation, range 5-348 months), and early-onset tumours ( $<12$ months after transplantation) occur in $<5 \%$ of cases [2]. Among PTSMT patients, $38 \%$ are children and the tumour occurs significantly earlier in juvenile patients [2]. In HIV-SMT, $<40 \%$ of patients are children [3-5]. In these juvenile cases, maternofetal HIV infection, infection after birth or duration of HIV infection cannot be linked to tumour manifestation [3-5]. Congenital immunodeficiency syndromes per se are very rare. Only a few CI-SMT cases have been described (all children) and in these cases at least, no association with a particular type of immune defect was evident, i.e. immunodeficiencies predisposing to severe EBV-associated complications like X-linked lymphoproliferative disease or CD27 deficiency do not seem to have an impact on CI-SMT development [6-11]. Furthermore, some patients with congenital immunodeficiency syndromes have undergone bone marrow transplantation and have then developed EBV-positive SMT (overlap between PTSMT and CI-SMT) [2, 12, 13].

As some PTSMT and HIV-SMT manifest near veins, e.g. in the cerebral sinus, the tumour founder cell is thought to be derived from an aberrant myogenous venous wall cell $[2,14]$. Almost all PTSMT and CI-SMT are EBV-positive while up to 30\% of HIV-SMT are EBV-negative (therefore, in these latter HIV-SMT cases, the proliferation cannot be linked to EBV) [2-11]. In lymphoid diseases, EBV infects B cells by using C3d/EBV receptor CD21 [1]. In most PTSMT, CD21 is not expressed, while HIV-SMT are frequently CD21-positive $[15,16]$. Therefore, the entry mechanism in most EBV-positive PTSMT is not clear. PTSMT express EBV proteins EBNA2/ EBNA3 but rarely latent membrane protein (LMP1), which corresponds to a variant of B cell latency type III; latency type III B cells are usually also positive for LMP1 $[1,2]$. In EBER-positive CI-SMT, similar to PTSMT, EBNA2 but not LMP1 and CD21 were detectable [6, 7]. It has been described that either the same EBV species or two different EBV species can lead to SMT and lymphoma in a single patient. In a case with PTSMT and PTLD, both tumour types showed the same 30-bp deletion in the LMP1 gene but TP53 mutation only in the PTLD and not the PTSMT, indicating that the same EBV species induced two unrelated tumours [17]. In contrast, in a patient with CI-SMT and a high-grade B cell lymphoma which were both EBV-positive, LMP1 deletion was present only in the CI-SMT and not in the unrelated lymphoma [8].

The molecular pathology of PTSMT and HIV-SMT is characterised by activated mTOR/Akt signal pathway $[18,19]$. Furthermore, in PTSMT, the EBV-related transcription factor v-myc myelocytomatosis viral oncogene homolog (avian; MYC) is increased when compared to EBV-negative sporadic leiomyomas [2]. Rearrangement of the MYC locus $8 \mathrm{q} 24$ could not be found; thus, it may be that other molecular mechanisms may lead to increased transcript expression [2]. Remarkably, several other EBV-related factors which are involved in cell cycle regulation, signal transduction, apoptosis and microRNA (miR-146a and miR-155) are not deregulated in PTSMT [2]. In CI-SMT, cytogenetic analysis showed no detectable aneuploidy or any other chromosomal defect [6].

\section{Clinical Characteristics and Diagnosis}

The clinical presentation of PTSMT, HIV-SMT and CI-SMT is unspecific (e.g. pain and organ dysfunction) and depends on the localisation and size of the tumour mass [2-11, 16]. Most PTSMT, HIV-SMT and CI-SMT are $<5 \mathrm{~cm}$ diameter but can be as big as $15 \mathrm{~cm}[18], 14 \mathrm{~cm}$ [5] or $21 \mathrm{~cm}$ [11], respectively. On magnetic resonance tomography (MRI), tumours may have characteristics of other mesenchymal neoplasms, e.g. intracranial meningioma [14]. An example of an intracranial PTSMT is shown in figure 1 . The main clinical differential diagnosis is immunosuppression-associated lymphoma, which can manifest at the same sites with similar unspecific symptoms. For example, in transplant patients, polymorphic or monomorphic B cell PTLD occur in a subgroup of patients (18\%) before PTSMT manifestation [2]. Corresponding to the general risk profile of PTLD, these patients with antecedent PTLD are mainly children [2]. Concomitant lymphomas are rarely reported in patients with HIV-SMT or CI-SMT $[8,20]$, while some HIVSMT patients suffer from simultaneous Kaposi sarcoma $[3-5,16]$.

All three tumour types can manifest at multiple sites. For example, one PTSMT patient has been described who suffered from $>80$ pulmonary tumours of a maximum di- 

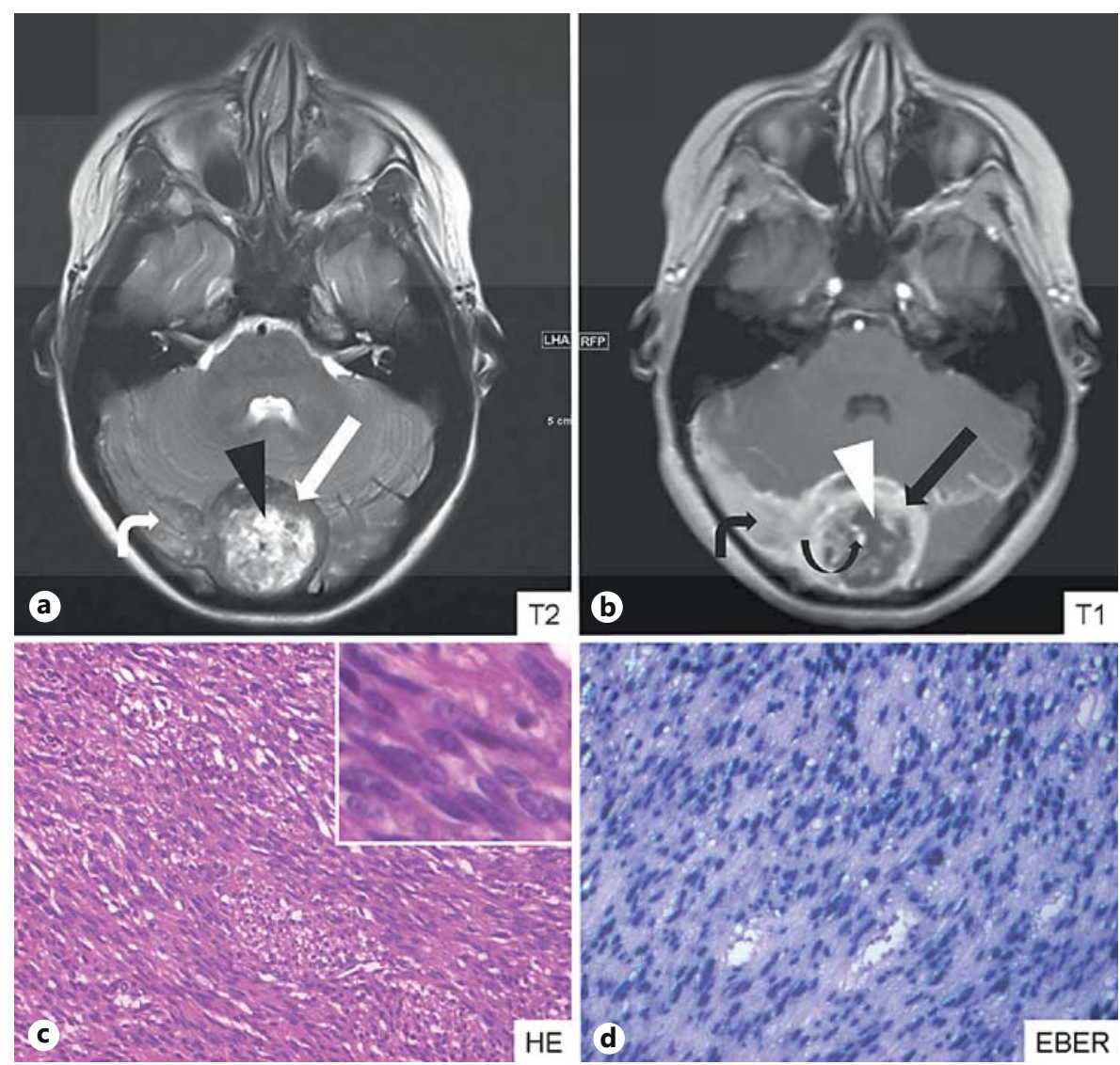

Fig. 1. Example of a PTSMT. a In this child (a 13-year-old girl), bone marrow transplantation was performed due to Fanconi anaemia. After 52 months, a cranial and a splenic PTSMT manifested. In this case, PTSMT were of recipient origin as could be evidenced by short tandem repeat. Cranial magnetic resonance tomography (MRI) of the posterior fossa shows a $4-\mathrm{cm}$ mass with signal characteristics of a solid margin (white arrow; T2-weighted image). No oedema in the adjuvant brain tissue is apparent. The inner parts of the tumour have hyperintense signals consistent with colliquation (black arrowhead). b A T1-weighted image at the identical position depicts strong enhancement of gadolinium-containing contrast media in the solid parts (black arrow in same position as in a). The hyperintense parts (a) do not enhance which is consistent with necrotic tissue (white arrowhead), i.e. only spots of enhancement are seen (curved black arrow). The solid parts infiltrate along the transverse sinus: angled arrows, white (a) and black (b). c Similar to other PTSMT, in this case the tumour tissue consists of intersecting fascicles of elongated spindle cells. Cells have an abundant eosinophilic cytoplasm positive for smooth muscle actin and desmin, elongated, partially box-shaped nuclei with coarse chromatin and only mild atypia with small nucleoli. Few cells have perinuclear vacuolisation and mitotic activity is low. Focal regressive changes such as hyalinisation, circumscribed necrosis, calcification and haemorrhage are evident. Some $\mathrm{T}$ cells are apparent. $\times 200$. Inset $\times 400$. $\mathbf{d}$ Nuclear EBER positivity can be found in most tumour cells. $\times 200$. ameter of $0.8 \mathrm{~cm}$ [21]. However, this does not necessarily define metastatic disease in all cases. At least in PTSMT, synchronous or metachronous tumours have been found and, in some patients, the tumour was shown to be of donor origin (presumably $<20 \%$ of cases) $[2,21]$. More than half the PTSMT develop in the liver/transplant liver, while in HIV-SMT and CI-SMT liver involvement is less frequent [2-11]. HIV-SMT appear to manifest more frequently in the vertebrae, central nervous system and skin $[3-5,16]$. In the few known CI-SMT cases, no preferential site of involvement can be identified [6-11]. Regarding sporadic SMT (EBV association is not expected in sporadic leiomyomas or leiomyosarcomas) which usually manifest in the uterus, uterine manifestation of PTSMT [22] is very rare and has not been reported in CI-SMT [6-11]. In HIV-positive adult female patients, uterine leiomyomas manifest in $16 \%$ of cases [23] but these are most likely sporadic. EBV-positive uterine HIV-SMT have not yet been reported. Diagnosis requires tumour sampling and subsequent histology, additional immuno- 
histology (positive for smooth muscle actin and desmin) and EBV detection in the tumour cells (EBER in situ hybridisation) [2]. There is only a loose (or non-existent?) correlation between the morphology and the biological behaviour of the tumour. This is certainly in contrast to conventional smooth muscle tumours, where the combination of location (uterus, skin, superficial and deep soft tissue compartments) and morphology (especially grading) can be used to predict the aggressiveness [24-26]. Due to the variable sites of PTSMT, HIV-SMT and CISMT manifestation, the localisation is not applicable as a criterion. Similar to sporadic SMT, these three tumours share spindle cell proliferation with eosinophil cytoplasm and elongated nucleoli (fig. 1) [2-11]. Spindle cells are arranged in a storiform pattern admixed with scattered small lymphocytes (mainly T cells). Smooth muscle markers are positive, while angiogenic markers (CD34, CD31, etc.) and KIT/CD117 are negative. The majority of PTSMT show no marked cellular atypia, very low to absent mitotic activity and no tumour necrosis [2]. Real 'sarcomalike' PTSMT with marked atypia and/or increased mitoses are rare. In contrast, in HIV-SMT, several different types can be distinguished: PTSMT-like variants (similar to leiomyoma or smooth muscle tumour of uncertain malignant potential), angioleiomyoma, myopericytomas and leiomyosarcoma with marked atypia [3]. The morphology of CI-SMT can be similar to PTSMT or may resemble leiomyosarcoma [5-11]. EBV association should be tested in all patients with any kind of immunosuppression and manifestation of a spindle cell proliferation. Serological analysis is not sufficient, because a positive result can be attributed to latent infection/unspecifically linked to immunosuppression, while EBV detection in tumour cells proves the EBV-associated proliferation in PTSMT, CISMT and in a subfraction of HIV-SMT. While EBV positivity is the best discriminator of sporadic SMT, the lack of EBV detection in HIV-SMT does not exclude the diagnosis [3]. Furthermore, except for the presence or absence of virus association, EBV-positive and EBV-negative HIV-SMT appear not to have morphological or clinical differences [3]. Without knowledge of the clinical context, establishment of definitive diagnosis with sub-classification into PTSMT, HIV-SMT or CI-SMT can be difficult.

In non-transplanted patients without an established diagnosis of HIV infection or congenital defects, SMT can be the first presentation $[3,11]$. Thus, manifestation of SMT in an unusual extra-uterine site, in particular after the detection of EBV association, should lead to further careful examination and evaluation of HIV infection (adults and children) and leukocyte defects (particularly in children).

\section{Therapy and Prognosis}

Due to the rarity of these tumours, our knowledge on effective therapy and prognosis is sparse and is derived from retrospective evaluations $[2,16]$. In general, most lesions appear to behave in a slow, locally progressive growth pattern and no definite criteria are established to predict the biological aggressiveness of PTSMT, HIVSMT or CI-SMT [25].

Surgical resection, if possible, should be performed, in particular, in symptomatic tumours. A retrospective meta-analysis revealed that in PTSMT, complete and incomplete resection or surgery with or without immunosuppressant showed no differences in prognosis [2]. There is evidence of a positive effect of sirolimus-based mTOR signal pathway inhibitor therapy in some cases, but due to contraindications, sirolimus cannot be administered to all patients $[27,28]$. Radiotherapy alone appears to be insufficient [2]. In some patients, reduced immunosuppression alone can lead to stable disease [2]. In addition, multiorgan involvement and/or transplant organ involvement and/or preceding PTLD and/or age have no impact on overall survival in PTSMT [2]. The only adverse prognostic factor, which could be identified in retrospective meta-analysis, is a cerebral manifestation of PTSMT [2]. In individual cases, the progressive growth of non-respectable tumours may result in an adverse clinical outcome. However, in general, in HIVSMT and CI-SMT but also in a subfraction of PTSMT, the prognosis appears to be related mainly to complications which are associated with immunosuppression and not to the tumour itself, e.g. bacterial pneumonia [2-11, 16]. Even a histological diagnosis of 'sarcoma' and/or large tumour masses does not appear to be an independent adverse prognostic factor and generally cannot be linked to prediction of clinical aggressiveness or tumourbiological behaviour $[2-11,16]$. Therefore, the term 'tumour' should be preferred for cases which do not or only partially meet the diagnostic criteria for conventional leiomyosarcoma.

\section{Summary}

PTSMT, HIV-SMT and CI-SMT represent rare and still poorly understood smooth muscle tumours. In contrast to sporadic SMT, all three types are associated with sustained immunosuppression and the majority of cases are EBV-positive. Tumours can manifest at any site and clinical presentation is unspecific. Surgery should be ap- 
plied in symptomatic cases, and, in PTSMT, reduced immunosuppression can lead to stable disease. In PTSMT, cerebral tumour manifestation is an additional adverse prognostic factor, but in general, the prognosis is mainly associated with co-morbidities and bacterial infection.

\section{Acknowledgements}

D.J. and F.L. received funding from the 'Integriertes Forschungs- und Behandlungszentrum Transplantation' (IFB-Tx) and the German Federal Ministry of Education and Research, reference No. 01EO0802, and B.M.-K. from the German Children's Cancer Fund and IFB-Tx.

\section{References}

1 Shroff R, Rees L: The post-transplant lymphoprolipherative disorder - a literature review. Pediatr Nephrol 2004;19:369-377.

-2 Jonigk D, Laenger F, Maegel L, Izykowski N, Rische J, Tiede C, Klein C, Maecker-Kolhoff B, Kreipe H, Hussein K: Molecular and clinicopathological analysis of Epstein-Barr virusassociated posttransplant smooth muscle tumors. Am J Transplant 2012;12:1908-1917.

-3 Ramdial PK, Sing Y, Deonarain J, Vaubell JI, Naicker S, Sydney C, Hadley LG, Singh B, Kiratu E, Gundry B, Sewram V: Extra-uterine myoid tumours in patients with acquired immunodeficiency syndrome: a clinicopathological reappraisal. Histopathology 2011;59: $1122-1134$

4 McClain KL, Leach CT, Jenson HB, Joshi VV, Pollock BH, Parmley RT, DiCarlo FJ, Chadwick EG, Murphy SB: Association of EpsteinBarr virus with leiomyosarcomas in children with AIDS. N Engl J Med 1995;332:12-18.

-5 Suankratay C, Shuangshoti S, Mutirangura A, Prasanthai V, Lerdlum S, Shuangshoti S, Pintong J, Wilde H: Epstein-Barr virus infectionassociated smooth-muscle tumors in patients with AIDS. Clin Infect Dis 2005;40:15211528.

6 6 Reyes C, Abuzaitoun O, De Jong A, Hanson C, Langston C: Epstein-Barr virus-associated smooth muscle tumors in ataxia-telangiectasia: a case report and review. Hum Pathol 2002;33:133-136.

7 Hatano M, Takada H, Nomura A, Ohga S, Ohshima K, Saeki I, Tajiri T, Taguchi T, Suita S, Hara T: Epstein-Barr virus-associated bronchial leiomyoma in a boy with cellular immunodeficiency. Pediatr Pulmonol 2006; 41:371-373.

8 Petrilli G, Lorenzi L, Paracchini R, Ubiali A, Schumacher RF, Cabassa P, Facchetti F: Epstein-Barr virus-associated adrenal smooth muscle tumors and disseminated diffuse large B-cell lymphoma in a child with common variable immunodeficiency: a case report and review of the literature. Int J Surg Pathol 2011, DOI: 10.1177/1066896911399901.

\9 Mierau GW, Greffe BS, Weeks DA: Primary leiomyosarcoma of brain in an adolescent with common variable immunodeficiency syndrome. Ultrastruct Pathol 1997;21:301305.

10 Tulbah A, Al-Dayel F, Fawaz I, Rosai J: Epstein-Barr virus-associated leiomyosarcoma of the thyroid in a child with congenital immunodeficiency: a case report. Am J Surg Pathol 1999;23:473-476.

11 Shaw RK, Issekutz AC, Fraser R, Schmit P, Morash B, Monaco-Shawver L, Orange JS, Fernandez CV: Bilateral adrenal EBV-associated smooth muscle tumors in a child with a natural killer cell deficiency. Blood 2012;119: 4009-4012.

12 Atluri S, Neville K, Davis M, Robertson KA, Marshalleck FE, O’Malley DP, Buckley RH, Nelson RP Jr: Epstein-Barr-associated leiomyomatosis and T-cell chimerism after haploidentical bone marrow transplantation for severe combined immunodeficiency disease. J Pediatr Hematol Oncol 2007;29:166-172.

13 Monforte-Muñoz H, Kapoor N, Saavedra JA: Epstein-Barr virus-associated leiomyomatosis and posttransplant lymphoproliferative disorder in a child with severe combined immunodeficiency: case report and review of the literature. Pediatr Dev Pathol 2003;6:449457.

14 Chaves NJ, Kotsimbos TC, Warren MA, McLean CA, Spelman DW, Williams TJ, Snell GI, Westall GP: Cranial leiomyosarcoma in an Epstein-Barr virus (EBV)-mismatched lung transplant recipient. J Heart Lung Transplant 2007;26:753-755.

15 Rougemont AL, Alfieri C, Fabre M, GorskaFlipot I, Papp E, Champagne J, Phan V, Fournet JC, Sartelet H: Atypical Epstein-Barr virus (EBV) latent protein expression in EBV-associated smooth muscle tumours occurring in paediatric transplant recipients. Histopathology 2008;53:363-367.

- 16 Purgina B, Rao UN, Miettinen M, Pantanowitz L: AIDS-related EBV-associated smooth muscle tumors: a review of 64 published cases. Patholog Res Int 2011;2011:561548.

17 Kingma DW, Shad A, Tsokos M, Fest T, Otsuki T, Frekko K, Werner E, Werner A, Magrath I, Raffeld M, Jaffe ES: Epstein-Barr virus (EBV)-associated smooth-muscle tumor arising in a post-transplant patient treated successfully for two PT-EBV-associated large-cell lymphomas. Case report. Am J Surg Pathol 1996;20:1511-1519.

18 Ong KW, Teo M, Lee V, Ong D, Lee A, Tan CS, Vathsala A, Toh HC: Expression of EBV latent antigens, mammalian target of rapamycin, and tumor suppression genes in EBVpositive smooth muscle tumors: clinical and therapeutic implications. Clin Cancer Res 2009; 15:5350-5358.

19 Shen Q, Feng W, Long MS, Duan X, Jaijakul $\mathrm{S}$, Arias CA, Brown RE, Zhao B: Multicentric hepatic EBV-associated smooth muscle tumors in an AIDS patient: a case report, investigation of mTOR activation and review of the literature. Int J Clin Exp Pathol 2011;4:421429.

20 Jimenez-Heffernan JA, Hardisson D, Palacios J, Garcia-Viera M, Gamallo C, Nistal M: Adrenal gland leiomyoma in a child with acquired immunodeficiency syndrome. Pediatr Pathol Lab Med 1995;15:923-929.

21 Somers GR, Tesoriero AA, Hartland E, Robertson CF, Robinson PJ, Venter DJ, Chow CW: Multiple leiomyosarcomas of both donor and recipient origin arising in a heartlung transplant patient. Am J Surg Pathol 1998;22:1423-1428.

22 Sunde J, Chetty-John S, Shlobin OA, Boice CR: Epstein-Barr virus-associated uterine leiomyosarcoma in an adult lung transplant patient. Obstet Gynecol 2010; 115:434-436.

23 Micheletti AR, Macedo AC, Silva GB, Silva AC, Silva-Vergara ML, Murta EF, Adad SJ: Benign and malignant neoplasias in 261 necropsies for HIV-positive patients in the period of 1989 to 2008. Rev Inst Med Trop Sao Paulo 2011;53:309-314.

24 LeBoit PE, Burg G, Weedon D, Sarasin A (ed): Pathology and Genetics of Tumours of the Skin. WHO Classification of Tumours. Lyon, IARC, 2005.

25 Fletcher CDM, Unni KK, Mertens F (eds): Pathology and Genetics of Tumours of Soft Tissue and Bone. WHO Classification of Tumours. Lyon, IARC, 2002.

26 Tavassoéli FA, Devilee P (eds): Pathology and Genetics of Tumours of the Breast and Female Genital Organs. WHO Classification of Tumours. Lyon, IARC, 2003.

27 Toh HC, Teo M, Ong KW, Lee V, Chan E, Lee AS, Vathsala A: Use of sirolimus for EpsteinBarr virus-positive smooth-muscle tumour. Lancet Oncol 2006;7:955-957.

28 Belingheri M, Comoli P, Locatelli F, Baldanti F, Martina V, Giani M, Ferraresso M, Cro L, Edefonti A, Ghio L: Successful medical treatment of EBV smooth muscle tumor in a renal transplant recipient. Pediatr Transplant 2010; 14:E101-E104. 